\title{
ON THE CLASSIFICATION OF ALMOST SQUARE-FREE MODULAR CATEGORIES
}

\author{
JINGCHENG DONG AND SONIA NATALE
}

\begin{abstract}
Let $\mathcal{C}$ be a modular category of Frobenius-Perron dimension $d q^{n}$, where $q>2$ is a prime number and $d$ is a square-free integer. We show that $\mathcal{C}$ must be integral and nilpotent and therefore grouptheoretical. In the case where $q=2$, we describe the structure of $\mathcal{C}$ in terms of equivariantizations of group-crossed braided fusion categories.
\end{abstract}

\section{INTRODUCTION}

Almost square-free (ASF) fusion categories are a class of fusion categories whose Frobenius-Perron dimensions factor simply. Recall that an ASF fusion category is a fusion category of Frobenius-Perron dimension $d q^{n}$, where $d$ is a square-free integer, $q$ is a prime number, $n$ is a non-negative integer and $\operatorname{gcd}(q, d)=1$.

We shall work over an algebraically closed field $k$ of characteristic zero. Recall that a modular category is a braided fusion category with a ribbon structure satisfying certain non-degeneracy condition (see Subsection 2.3 for a precise definition of this notion). Modular categories are a relevant class of fusion categories due to their applications in areas like low dimensional topology and conformal field theory.

The problem of classifying ASF modular categories has been addressed in several papers. It was shown in 11 that every braided fusion category of Frobenius-Perron dimension $q^{n}$ is group-theoretical. The second-named author showed in [29, Corollary 7.3] that every ASF modular category is solvable. For distinct prime numbers $q>2, p$ and $r$, results on the structure of modular categories of Frobenius-Perron dimensions $p q^{n}, p q r, 4 d$ and $8 d$, where $1 \leq n \leq 5$ and $d$ is an odd natural number, were obtained in [14, 26, 1, 3, 9, 10.

In the present paper our first main result gives a nearly complete classification of these modular categories. We prove the following theorem:

Date: November 1, 2017.

2010 Mathematics Subject Classification. 18D10; $16 \mathrm{~T} 05$.

Key words and phrases. Braided fusion category; modular category; group-theoretical fusion category; braided $G$-crossed fusion category; Tannakian category. 
Theorem 4.7. Let $q>2$ be a prime number and let $d$ be a square-free integer. Suppose that $\mathcal{C}$ is a modular category such that $\operatorname{FPdim} \mathcal{C}=d q^{n}$, $n \geq 0$. Then $\mathcal{C}$ is integral and nilpotent.

As an application of Theorem 4.7 we show that an ASF modular category of Frobenius-Perron dimension $d q^{4}$, where $q$ is a odd prime number not dividing $d$ is necessarily pointed. See Corollary 4.13.

By [11], every integral nilpotent braided fusion category is group-theoretical. Therefore Theorem 4.7 implies that any ASF modular category of the specified dimension, namely, except in the $q=2$ integral case, is grouptheoretical, entailing the classification of such modular categories in grouptheoretical terms, as explained below.

Group-theoretical fusion categories are important examples of fusion categories whose objects have integer Frobenius-Perron dimensions. They are also interesting examples of fusion categories that can be described explicitly in terms of finite groups and their cohomology [15.

Let $G$ be a finite group, $H \leq G$ a subgroup of $G, \omega: G \times G \times G \rightarrow k^{\times}$ a normalized 3-cocycle, and $\psi: H \times H \rightarrow k^{\times}$a normalized 2-cochain such that $\mathrm{d} \psi=\left.\omega\right|_{H}$. Consider the fusion category $\operatorname{Vect}_{G}^{\omega}$ of finite dimensional $G$-graded vector spaces with associativity given by 3-cocycle $\omega$. Then the twisted group algebra $k_{\psi}[H]$ is an associative algebra in $\operatorname{Vect}_{G}^{\omega}$. Thus the category $\mathcal{C}(G, \omega, H, \psi)$ of $k_{\psi}[H]$-bimodules in $\operatorname{Vect}_{G}^{\omega}$ is a fusion category with tensor product $\otimes_{k_{\psi}[H]}$ and unit object $k_{\psi}[H]$. A fusion category is called group-theoretical if it is equivalent to one of the form $\mathcal{C}(G, \omega, H, \psi)$ [15, Section 8.8].

Let $\mathcal{C}$ be a braided group-theoretical fusion category. The braiding of $\mathcal{C}$ yields a canonical embedding of $\mathcal{C}$ into its Drinfeld center $\mathcal{Z}(\mathcal{C})$. Following the proof of [27, Theorem 1.2], $\mathcal{Z}(\mathcal{C})$ is equivalent to $\mathcal{Z}\left(\operatorname{Vect}_{G}^{\omega}\right) \cong \operatorname{Rep}\left(D^{\omega} G\right)$ for some finite group $G$ and 3-cocycle $\omega$ on $G$, where $\operatorname{Rep}\left(D^{\omega} G\right)$ is the representation category of the twisted quantum double $D^{\omega} G$ [7]. Therefore, every braided group-theoretical fusion category may be realized as a fusion subcategory of $\operatorname{Rep}\left(D^{\omega} G\right)$. One of the main results of [25] describes all fusion subcategories of $\operatorname{Rep}\left(D^{\omega} G\right)$. Therefore this result gives a complete description of all braided group-theoretical fusion categories in group-theoretical terms. In the light of these observations, (braided) group-theoretical fusion categories are well understood.

An approach towards an explicit parameterization of the modular categories in Theorem 4.7 is discussed in Remark 4.9.

If $\mathcal{C}$ is an integral ASF modular category of dimension $d q^{n}$ then $\mathcal{C}$ can be obtained by a $G$-equivariantization from a nilpotent fusion category of nilpotency class 2 [9], where $G$ is a $q$-group. Our second task of this paper is to describe the structure of $\mathcal{C}$ when $\mathcal{C}$ is strictly weakly integral; that is, when there exists at least a simple object $X$ such that FPdim $X$ is not an integer. 
Suppose that $\mathcal{C}$ is a modular category containing a Tannakian subcategory $\mathcal{E} \cong \operatorname{Rep} G$, where $G$ is a finite group. Then $\mathcal{C}$ can be obtained as a $G$-equivariantization of a braided $G$-crossed fusion category $\oplus_{g \in G} \mathcal{D}_{g}$; see Subsection 2.4 for a discussion of this fact.

Our second main result is the following theorem.

Theorem 5.6. Let $\mathcal{C}$ be a strictly weakly integral ASF modular category. Then $\mathcal{C}$ fits into one of the following classes:

(1) $\mathcal{C}$ is equivalent to a Deligne tensor product $\mathcal{I} \otimes \mathcal{B}$, where $\mathcal{I}$ is an Ising fusion category and $\mathcal{B}$ is a pointed modular category.

(2) $\mathcal{C}$ is equivalent to a $G$-equivariantization of a braided $G$-crossed fusion category $\oplus_{g \in G} \mathcal{D}_{g}$, where $G$ is a 2 -group and $\mathcal{D}_{e}$ is a pointed modular category.

(3) $\mathcal{C}$ is equivalent to a $G$-equivariantization of a braided $G$-crossed fusion category $\oplus_{g \in G} \mathcal{D}_{g}$, where $G$ is a 2 -group and $\mathcal{D}_{e} \cong \mathcal{I} \otimes \mathcal{B}$, where $\mathcal{I}$ is an Ising fusion category and $\mathcal{B}$ is a pointed modular category.

Recall that an Ising braided category is a non-pointed braided fusion category of Frobenius-Perron dimension 4. Every Ising braided category is non-degenerate. See [12, Appendix B] for a classification of Ising braided categories.

This paper is organized as follows. In Section 2, we recall some basic results and prove some basic lemmas which will be used throughout. In Section 3, we determine an upper bound for the order of the dimensional grading group of a weakly integral modular category. This result will be used in Sections 4 and 5. In Section 4, we study the nilpotency of an integral ASF modular category and give a proof of Theorem 4.7. In Section 5, we study the structure of a strictly weakly integral ASF modular category and give a proof of Theorem 5.6.

\section{Preliminaries}

The category of finite dimensional vector spaces over $k$ will be denoted by Vect. All tensor categories will be assumed to be strict, unless explicitly stated. We refer the reader to [15], [16], [12] for the notions on fusion categories and braided fusion categories used throughout.

2.1. Frobenius-Perron dimension. Let $\mathcal{C}$ be a fusion category. The Frobenius-Perron dimension FPdim $X$ of a simple object $X \in \mathcal{C}$ is defined as the Frobenius-Perron eigenvalue of the matrix of left multiplication by the class of $X$ in the basis $\operatorname{Irr}(\mathcal{C})$ of the Grothendieck ring of $\mathcal{C}$ consisting of isomorphism classes of simple objects. The Frobenius-Perron dimension of $\mathcal{C}$ is $\mathrm{FPdim} \mathcal{C}=\sum_{X \in \operatorname{Irr}(\mathcal{C})}(\mathrm{FPdim} X)^{2}$. The fusion category $\mathcal{C}$ is called integral if $\mathrm{FPdim} X$ is a natural number, for all simple object $X \in \mathcal{C}$, and it is called weakly integral if $\mathrm{FPdim} \mathcal{C}$ is a natural number. We shall say that $\mathcal{C}$ is strictly weakly integral if it is weakly integral but not integral. 
2.2. Nilpotency of a fusion category. Let $G$ be a finite group and let $\mathcal{C}$ be a fusion category. A $G$-grading on a fusion category $\mathcal{C}$ is a decomposition $\mathcal{C}=\oplus_{g \in G} \mathcal{C}_{g}$, such that $\mathcal{C}_{g} \otimes \mathcal{C}_{h} \subseteq \mathcal{C}_{g h}$ and $\mathcal{C}_{g}^{*} \subseteq \mathcal{C}_{g^{-1}}$, for all $g, h \in G$. The fusion category $\mathcal{C}$ is called a $G$-extension of a fusion category $\mathcal{D}$ if there is a faithful grading $\mathcal{C}=\oplus_{g \in G} \mathcal{C}_{g}$ with neutral component $\mathcal{C}_{e} \cong \mathcal{D}$.

If $\mathcal{C}$ is any fusion category, there exists a finite group $\mathcal{U}(\mathcal{C})$, called the universal grading group of $\mathcal{C}$, and a canonical faithful grading $\mathcal{C}=\oplus_{g \in \mathcal{U}(\mathcal{C})} \mathcal{C}_{g}$, with neutral component $\mathcal{C}_{e}=\mathcal{C}_{a d}$, where $\mathcal{C}_{a d}$ is the adjoint subcategory of $\mathcal{C}$, that is, the fusion subcategory generated by $X \otimes X^{*}$, where $X$ runs over the simple objects of $\mathcal{C}$.

The descending central series of $\mathcal{C}$ is the series of fusion subcategories

$$
\cdots \subseteq \mathcal{C}^{(n+1)} \subseteq \mathcal{C}^{(n)} \subseteq \cdots \subseteq \mathcal{C}^{(1)} \subseteq \mathcal{C}^{(0)}=\mathcal{C},
$$

where, for each $n \geq 0, \mathcal{C}^{(n+1)}=\left(\mathcal{C}^{(n)}\right)_{a d}$.

The fusion category $\mathcal{C}$ is nilpotent if there exists $n \geq 0$ such that $\mathcal{C}^{(n)} \cong$ Vect. Equivalently, $\mathcal{C}$ is nilpotent if there exist a sequence of fusion categories Vect $=\mathcal{C}_{0} \subseteq \mathcal{C}_{1} \cdots \subseteq \mathcal{C}_{n}=\mathcal{C}$, and a sequence of finite groups $G_{1}, \ldots, G_{n}$, such that for all $i=1, \ldots, n, \mathcal{C}_{i}$ is a $G_{i}$-extension of $\mathcal{C}_{i-1}$.

2.3. Braided fusion categories. A braided fusion category is a fusion category endowed with a braiding, that is, a natural isomorphism $c_{X, Y}$ : $X \otimes Y \rightarrow Y \otimes X, X, Y \in \mathcal{C}$, subject to the hexagon axioms.

Let $\mathcal{C}$ be a braided fusion category. If $\mathcal{D}$ is a fusion subcategory of $\mathcal{C}$, the Müger centralizer $\mathcal{D}^{\prime}$ of $\mathcal{D}$ in $\mathcal{C}$ is the full fusion subcategory generated by objects $X \in \mathcal{C}$ such that $c_{Y, X} c_{X, Y}=\operatorname{id}_{X \otimes Y}$, for all objects $Y \in \mathcal{D}$.

The Müger (or symmetric) center of $\mathcal{C}$ is the Müger centralizer $\mathcal{C}^{\prime}$. The category $\mathcal{C}$ is called symmetric if $\mathcal{C}^{\prime}=\mathcal{C}$.

Let $G$ be a finite group. The fusion category $\operatorname{Rep} G$ of finite dimensional representations of $G$ is a symmetric fusion category with respect to the canonical braiding. A braided fusion category $\mathcal{E}$ is called Tannakian, if $\mathcal{E} \cong \operatorname{Rep} G$ for some finite group $G$, as symmetric fusion categories.

Every symmetric fusion category $\mathcal{C}$ is super-Tannakian, that is, there exist a finite group $G$ and a central element $u \in G$ of order 2 , such that $\mathcal{C}$ is equivalent to the category $\operatorname{Rep}(G, u)$ of representations of $G$ on finitedimensional super-vector spaces where $u$ acts as the parity operator.

Hence if $\mathcal{C} \cong \operatorname{Rep}(G, u)$ is a symmetric fusion category, then $\mathcal{E}=\operatorname{Rep} G / u$ is the unique Tannakian subcategory of $\mathcal{C} \operatorname{such}$ that $\mathrm{FPdim} \mathcal{E}=\mathrm{FPdim} \mathcal{C} / 2$. Thus if FPdim $\mathcal{C}$ is bigger than 2, then $\mathcal{C}$ necessarily contains a Tannakian subcategory, and a non-Tannakian symmetric fusion category of FrobeniusPerron dimension 2 is equivalent to the category sVect of finite-dimensional super-vector spaces. See [12, Subsection 2.12].

If $\mathcal{C}$ is any braided fusion category, its Müger center $\mathcal{C}^{\prime}$ is a symmetric fusion subcategory of $\mathcal{C}$. $\mathcal{C}$ is called non-degenerate (respectively, slightly degenerate) if $\mathcal{C}^{\prime} \cong$ Vect (respectively, if $\mathcal{C}^{\prime} \cong$ sVect). A modular category 
is a non- degenerate braided fusion category with a ribbon structure. By [15. Proposition 8.23, Proposition 8.24], a weakly integral braided fusion category is modular if and only if it is non-degenerate.

Suppose $\mathcal{C}$ is a braided nilpotent fusion category. It is shown in [11, Theorem 1.1] that $\mathcal{C}$ admits a unique decomposition into a tensor product of braided fusion categories

$$
\mathcal{C}_{p_{1}} \otimes \cdots \otimes \mathcal{C}_{p_{n}}
$$

where for all $1 \leq i \leq n, p_{i}$ is a prime number such that the Frobenius-Perron dimension of $\mathcal{C}_{p_{i}}$ is a power of $p_{i}$ and the primes $p_{1}, \ldots, p_{n}$ are pairwise distinct.

Remark 2.1. Consider the decomposition (2.2). If $\mathcal{C}$ is non-degenerate, then the categories $\mathcal{C}_{p_{1}}, \ldots, \mathcal{C}_{p_{n}}$ are non-degenerate as well. In fact, for all $1 \leq i \leq n$, we have $\mathrm{FP} \operatorname{dim} \mathcal{C}=\mathrm{FP} \operatorname{dim} \mathcal{C}_{p_{i}} \mathrm{FP} \operatorname{dim} \mathcal{C}_{p_{i}}^{\prime}$. Therefore $\mathrm{FPdim} \mathcal{C}_{p_{i}}$ and $\mathrm{FP} \operatorname{dim} \mathcal{C}_{p_{i}}^{\prime}$ are relatively prime. Hence the Müger center of $\mathcal{C}_{p_{i}}$, which coincides with $\mathcal{C}_{p_{i}} \cap \mathcal{C}_{p_{i}}^{\prime}$ must be trivial, that is, $\mathcal{C}_{p_{i}}$ is non-degenerate.

We next prove some results that will be needed in the course of the proof of Theorem 4.7 .

Lemma 2.2. Let $\mathcal{C}_{1}, \mathcal{C}_{2}$ be fusion subcategories of a braided fusion category $\mathcal{C}$ and let $\mathcal{C}_{1} \vee \mathcal{C}_{2}$ be the fusion subcategory generated by $\mathcal{C}_{1}$ and $\mathcal{C}_{2}$. Then $\left(\mathcal{C}_{1} \vee \mathcal{C}_{2}\right)^{(n)}=\mathcal{C}_{1}^{(n)} \vee \mathcal{C}_{2}^{(n)}$, for all $n \geq 0$.

Proof. Observe that if a fusion category $\mathcal{D}$ is generated by objects $X_{1}, \ldots, X_{r}$, $r \geq 1$, then $\mathcal{D}_{a d}$ is generated by $X_{1} \otimes X_{1}^{*}, \ldots, X_{r} \otimes X_{r}^{*}$. This implies that the adjoint subcategory of $\mathcal{C}_{1} \vee \mathcal{C}_{2}$ coincides with the fusion subcategory $\left(\mathcal{C}_{1}\right)_{a d} \vee\left(\mathcal{C}_{2}\right)_{a d}$ generated by $\left(\mathcal{C}_{1}\right)_{a d}$ and $\left(\mathcal{C}_{2}\right)_{a d}$. The lemma follows by induction on $n \geq 0$.

Suppose that $\mathcal{C}$ is a fusion category such that the Grothendieck ring $\mathrm{K}_{0}(\mathcal{C})$ is commutative (e.g. if $\mathcal{C}$ is braided). Let $\mathcal{D}$ be a fusion subcategory of $\mathcal{C}$. Recall that the commutator $\mathcal{D}^{c o}$ is a fusion subcategory of $\mathcal{C}$, where $\mathcal{D}^{c o}$ is the fusion subcategory of $\mathcal{C}$ generated by all objects $X$ such that $X \otimes X^{*}$ is an object of $\mathcal{D}$. It follows from [17, Lemma 4.15] that $\left(\mathcal{D}^{c o}\right)_{a d} \subseteq \mathcal{D} \subseteq\left(\mathcal{D}_{a d}\right)^{c o}$.

Proposition 2.3. Let $\mathcal{C}$ be a braided fusion category. Then the following hold:

(i) $\mathcal{C}$ contains a unique maximal nilpotent fusion subcategory $\mathcal{C}_{\text {nil }}$.

(ii) Suppose $\mathcal{C}$ is non-degenerate. Let $\mathcal{D} \subseteq \mathcal{C}$ be the Müger centralizer of the fusion subcategory $\mathcal{C}_{\text {nil }}$. Then $\mathcal{D}_{a d}=\mathcal{D}$, in other words, $\mathcal{U}(\mathcal{D})=1$.

Proof. Suppose that $\mathcal{C}_{1}$ and $\mathcal{C}_{2}$ are fusion subcategories of $\mathcal{C}$ such that $\mathcal{C}_{1}$ and $\mathcal{C}_{2}$ are nilpotent. It follows from Lemma 2.2 that the fusion subcategory $\mathcal{C}_{1} \vee \mathcal{C}_{2}$ generated by $\mathcal{C}_{1}$ and $\mathcal{C}_{2}$ is nilpotent. Hence the fusion subcategory $\mathcal{C}_{\text {nil }}$ generated by all nilpotent fusion subcategories of $\mathcal{C}$ is nilpotent. This proves (i). 
Now assume that $\mathcal{C}$ is non-degenerate. By [12, Proposition 3.25] we have that $\left(\mathcal{D}_{a d}\right)^{\prime}=\left(\mathcal{D}^{\prime}\right)^{c o}$. Since $\mathcal{C}$ is non-degenerate, then $\mathcal{D}^{\prime}=\mathcal{C}_{\text {nil }}^{\prime \prime}=\mathcal{C}_{\text {nil }}$ [12, Theorem 3.10], so that $\left(\mathcal{D}_{a d}\right)^{\prime}=\left(\mathcal{C}_{\text {nil }}\right)^{c o}$.

On the other hand, $\left(\left(\mathcal{C}_{n i l}\right)^{c o}\right)_{a d} \subseteq \mathcal{C}_{\text {nil }}$, hence $\left(\mathcal{C}_{n i l}\right)^{c o}$ is nilpotent. Since $\mathcal{C}_{\text {nil }} \subseteq\left(\mathcal{C}_{\text {nil }}\right)^{c o}$, we obtain that $\mathcal{C}_{\text {nil }}=\left(\mathcal{C}_{\text {nil }}\right)^{c o}$, by maximality of $\mathcal{C}_{\text {nil }}$. Then $\left(\mathcal{D}_{a d}\right)^{\prime}=\mathcal{C}_{n i l}$ and therefore $\mathcal{D}_{a d}=\left(\mathcal{D}_{a d}\right)^{\prime \prime}=\left(\mathcal{C}_{n i l}\right)^{\prime}=\mathcal{D}$. This proves part (ii) and finishes the proof of the proposition.

\subsection{Braided $G$-crossed fusion categories and Tannakian subcate-} gories of a braided fusion category. Let $G$ be a finite group. Let us recall the correspondence between equivalence classes of braided fusion categories containing $\operatorname{Rep} G$ as a Tannakian subcategory and equivalence classes of braided $G$-crossed fusion categories [24, [12, Section 4.4].

A braided $G$-crossed fusion category is a fusion category $\mathcal{C}$ endowed with a $G$-grading $\mathcal{C}=\oplus_{g \in G} \mathcal{C}_{g}$ and an action of $G$ by tensor autoequivalences $\rho: \underline{G} \rightarrow$ Aut $_{\otimes} \mathcal{C}$, such that $\rho^{g}\left(\mathcal{C}_{h}\right) \subseteq \mathcal{C}_{g h g^{-1}}$, for all $g, h \in G$, and a $G$ braiding $c: X \otimes Y \rightarrow \rho^{g}(Y) \otimes X, g \in G, X \in \mathcal{C}_{g}, Y \in \mathcal{C}$, subject to appropriate compatibility conditions.

Every $G$-crossed braided fusion category gives rise, through the equivariantization process, to a braided fusion category containing $\operatorname{Rep} G$ as a Tannakian subcategory. Conversely, suppose that $\mathcal{E} \cong \operatorname{Rep} G$ is a Tannakian subcategory of a braided fusion category $\mathcal{C}$. Then the de-equivariantization $\mathcal{C}_{G}$ of $\mathcal{C}$ with respect to $\mathcal{E}$ is a braided $G$-crossed fusion category in a canonical way.

The neutral component $\mathcal{C}_{G}^{0}$ of $\mathcal{C}_{G}$ with respect to the associated $G$-grading is a braided fusion category and the crossed action of $G$ on $\mathcal{C}_{G}$ induces an action of $G$ on $\mathcal{C}_{G}^{0}$ by braided auto-equivalences. Moreover, there is an equivalence of braided fusion categories $\left(\mathcal{C}_{G}^{0}\right)^{G} \cong \mathcal{E}^{\prime}$, where $\mathcal{E}^{\prime}$ is the centralizer in $\mathcal{C}$ of the Tannakian subcategory $\mathcal{E}$.

The braided fusion category $\mathcal{C}$ is non-degenerate if and only if the neutral component $\mathcal{C}_{G}^{0}$ of $\mathcal{C}_{G}$ is non-degenerate and the $G$-grading of $\mathcal{C}_{G}$ is faithful 12, Proposition 4.6 (ii)]. In particular, if $\mathcal{C}$ is a non-degenerate braided fusion category containing a Tannakian subcategory $\mathcal{E} \cong \operatorname{Rep} G$, then $|G|^{2}$ divides $\operatorname{FPdim} \mathcal{C}$.

We shall use the following result:

Theorem 2.4. [9, Theorem 4.1]. Let $q$ be a prime number and let $d$ be a square-free natural number. Let also $\mathcal{C}$ be an integral modular category such that $\mathrm{FP} \operatorname{dim} \mathcal{C}=d q^{n}, n \geq 0$. Suppose that $\mathcal{E} \subseteq \mathcal{C}$ is a maximal Tannakian subcategory. Then $\mathcal{E}^{\prime}$ is group-theoretical.

Note that if $\mathcal{E} \cong \operatorname{Rep} G$ is a maximal Tannakian subcategory, then $\mathcal{E}^{\prime} \cong$ $\left(\mathcal{C}_{G}^{0}\right)^{G}$, where $\mathcal{C}_{G}^{0}$ is the core of $\mathcal{C}$ introduced in [12, Section 5]. In fact, it is shown in 9 that the core of $\mathcal{C}$ is a pointed non-degenerate braided fusion category. 


\section{THE ORDER OF THE DIMENSIONAL GRADING GROUP OF A WEAKLY INTEGRAL MODULAR CATEGORY}

Let $\mathcal{C}$ be a weakly integral fusion category. It was shown in [17 that $\mathcal{C}=$ $\oplus_{g \in E} \mathcal{C}_{g}$ is faithfully graded by an elementary abelian 2-group $E$. Moreover, there is a set of distinct square-free integers $n_{g}, g \in E$, such that $n_{e}=1$ and FPdim $X \in \mathbb{Z} \sqrt{n_{g}}$, for every $X \in \operatorname{Irr}\left(\mathcal{C}_{g}\right)$. This canonical grading is called the dimensional grading of $\mathcal{C}$. The neutral component $\mathcal{C}_{e}$ of this grading is denoted by $\mathcal{C}_{\text {int }}$. The group $E$ will be called the dimensional grading group of $\mathcal{C}$.

The Frobenius-Perron dimension of every weakly integral fusion category has the form $d 2^{n}$, where $n$ is a non-negative integer and $d$ is an odd natural number. The following theorem gives an upper bound for the order of $E$ in the case where $\mathcal{C}$ is a modular category.

Theorem 3.1. Let $\mathcal{C}$ be a weakly integral modular category and let $\mathcal{C}=$ $\oplus_{g \in E} \mathcal{C}_{g}$ be its dimensional grading. Suppose that FPdim $\mathcal{C}=d 2^{n}$, where $n \geq 0$ and $d$ is an odd natural number. Then $|E| \leq 2^{\frac{n}{2}}$. In other words, $|E|^{2}$ divides the Frobenius-Perron dimension of $\mathcal{C}$.

Proof. The neutral component $\mathcal{C}_{\text {int }}$ is the unique component of this grading whose objects are integral, that is, $\mathrm{FPdim} X \in \mathbb{Z}$ for every $X \in \mathcal{C}_{\text {int }}$. Hence, the largest pointed fusion subcategory $\mathcal{C}_{p t}$ is a fusion subcategory of $\mathcal{C}_{\text {int }}$ and therefore $\mathrm{FPdim} \mathcal{C}_{p t} \operatorname{divides} \mathrm{FPdim} \mathcal{C}_{\text {int }}$, by [15, Proposition 8.15]. On the other hand, FPdim $\mathcal{C}_{p t}=|\mathcal{U}(\mathcal{C})|$ by [17, Theorem 6.2] and hence $|\mathcal{U}(\mathcal{C})|$ divides FPdim $\mathcal{C}_{\text {int }}$. In addition, [17, Corollary 3.7] shows that there is a surjective group homomorphism $\pi: \mathcal{U}(\mathcal{C}) \rightarrow E$. Hence $|E|$ divides $|\mathcal{U}(\mathcal{C})|$.

Since $E$ is an elementary abelian 2-group, we may assume that $|E|=2^{t}$ for some non-negative integer $t$. From the arguments above, we have

$\operatorname{FPdim} \mathcal{C}_{\text {int }}=|\mathcal{U}(\mathcal{C})| m_{2}, 2^{t} m_{1}=|\mathcal{U}(\mathcal{C})|$ and $2^{t} \mathrm{FPdim} \mathcal{C}_{\text {int }}=\mathrm{FPdim} \mathcal{C}$, where $m_{1}, m_{2}$ are positive integers. These equalities imply that $2^{n-2 t} d=$ $m_{1} m_{2}$. Hence we get that $2^{t}=|E| \leq 2^{\frac{n}{2}}$.

Corollary 3.2. Let $\mathcal{C}$ be a weakly integral braided fusion category such that FPdim $\mathcal{C}$ is not divisible by 4. Then $\mathcal{C}$ is integral.

In other words, if $\mathcal{C}$ is a strictly weakly integral braided fusion category then 4 divides FPdim $\mathcal{C}$.

Proof. We may assume that $\mathrm{FPdim} \mathcal{C}=d 2^{n}$, where $d$ is odd and $n=0$ or 1. If $n=0$ then FPdim $\mathcal{C}$ is odd, and hence $\mathcal{C}$ is automatically integral by [17. Corollary 3.11]. If $n=1$ and $\mathcal{C}$ is non-degenerate then the order of $E$ is 1 by Theorem 3.1. This means that $\mathcal{C}=\mathcal{C}_{\text {int }}$ is integral. In the rest of our proof, we shall consider the case when $n=1$ and $\mathcal{C}$ is degenerate.

Suppose on the contrary that $\mathcal{C}$ is strictly weakly integral. In this case [17. Theorem 3.10] shows that $\mathcal{C}$ has a faithful $G$-grading, where $G$ is an elementary abelian 2-group. Since $2 \mid F P \operatorname{dim} \mathcal{C}$ and $4 \nmid F P \operatorname{dim} \mathcal{C}$, we have 
$G \cong \mathbb{Z}_{2}$ and hence $\mathcal{C}=\mathcal{C}_{0} \oplus \mathcal{C}_{1}$. In addition, [17, Theorem 3.10] also shows that $X \in \operatorname{Irr}(\mathcal{C})$ is integral if and only if $X \in \mathcal{C}_{0}$. Notice that FPdim $\mathcal{C}_{0}=$ $\mathrm{FP} \operatorname{dim} \mathcal{C} / 2$ is an odd integer.

Let $\mathcal{E}$ be the Müger center of $\mathcal{C}$. It is not trivial by assumption. Since $\mathcal{E}$ is symmetric, then it is integral and therefore $\mathcal{E} \subseteq \mathcal{C}_{0}$. Thus FPdim $\mathcal{E}$ must be odd, whence $\mathcal{E} \cong \operatorname{Rep}(H)$ is a Tannakian subcategory of $\mathcal{C}$ for some finite group $H$. We may thus consider the de-equivariantization $\mathcal{C}_{H}$ of $\mathcal{C}$ by $\operatorname{Rep}(H)$, which is a non-degenerate braided fusion category.

Observe that $\mathcal{C}_{H}$ is weakly integral and $\mathrm{FP} \operatorname{dim} \mathcal{C}=|H| \mathrm{FPdim} \mathcal{C}_{H}$, so that FPdim $\mathcal{C}_{H}$ is not divisible by 4 . It follows from the first part of the proof that $\mathcal{C}_{H}$ is integral. Then $\mathcal{C}$ is integral because the class of integral fusion categories is closed under taking equivariantization [4]. This completes the proof of the corollary.

Remark 3.3. If 4 divides $\mathrm{FP} \operatorname{dim} \mathcal{C}$, then $\mathcal{C}$ may be not integral. An example of this class of modular categories is classified in [2].

On the other hand, Corollary 3.2 combined with [13, Lemma 1.2] implies that a weakly integral modular category such that $\mathrm{FPdim} \mathcal{C}$ is square-free is necessarily pointed; see [2, Lemma 3.4].

\section{Nilpotency of A ClASS OF ASF MOdular CATEGORIES}

Let $q$ be a prime number and let $d$ be a square-free natural number not divisible by $q$. Throughout this section $\mathcal{C}$ will denote an ASF modular category of Frobenius-Perron dimension $d q^{n}$, unless otherwise stated.

Recall from Proposition 2.3 that $\mathcal{C}$ contains a unique maximal nilpotent fusion subcategory $\mathcal{C}_{\text {nil }}$. In what follows we shall denote by $\mathcal{D}=\mathcal{C}_{\text {nil }}^{\prime}$ the centralizer of $\mathcal{C}_{\text {nil }}$. By Proposition 2.3, $\mathcal{D}_{a d}=\mathcal{D}$.

Since $\mathcal{C}$ is modular, then [12, Theorem 3.14] implies that

$$
\mathrm{FP}_{\operatorname{dim}} \mathcal{C}_{\text {nil }}=a q^{m}, \quad \mathrm{FP} \operatorname{dim} \mathcal{D}=b q^{n-m},
$$

where $a$ and $b$ are natural numbers such that $a b=d$, and $1 \leq m \leq n$.

Remark 4.1. Observe that if $\mathcal{E}$ is a Tannakian subcategory of $\mathcal{C}$ then $(\mathrm{FPdim} \mathcal{E})^{2}$ divides $\mathrm{FPdim} \mathcal{C}$. Hence $\mathrm{FPdim} \mathcal{E}$ is a power of $q$. In particular, every Tannakian subcategory of $\mathcal{C}$ is nilpotent and therefore it is contained in $\mathcal{C}_{\text {nil }}$.

In the next lemmas we assume that $\mathcal{C}$ is integral.

Lemma 4.2. The categories $\mathcal{C}_{\text {nil }}$ and $\mathcal{D}$ are group-theoretical.

Proof. Since $\mathcal{C}_{n i l}$ is nilpotent and integral, then it is group-theoretical [11]. Let $\mathcal{E} \subseteq \mathcal{C}$ be a maximal Tannakian subcategory. It follows from Theorem 2.4 that $\mathcal{E}^{\prime}$ is group-theoretical.

As observed in Remark 4.1, $\mathcal{E} \subseteq \mathcal{C}_{\text {nil }}$. Then $\mathcal{D}=\left(\mathcal{C}_{\text {nil }}\right)^{\prime} \subseteq \mathcal{E}^{\prime}$ and therefore $\mathcal{D}$ is also group-theoretical, as claimed.

Since $\mathcal{D}$ is a group-theoretical braided fusion category, it follows from 25 , Theorem 7.2] that $\mathcal{D}$ contains a Tannakian subcategory $\mathcal{E} \cong \operatorname{Rep} G$ such that the de-equivariantization $\mathcal{D}_{G}$ is a pointed fusion category. 
Lemma 4.3. Let $\mathcal{E} \cong \operatorname{Rep} G$ be a Tannakian subcategory of $\mathcal{D}$ such that $\mathcal{D}_{G}$ is pointed. Then $\mathcal{E}$ is contained in the Müger center of $\mathcal{D}$. In particular, $\mathcal{D}_{G}$ is a braided fusion category and the canonical dominant functor $F: \mathcal{D} \rightarrow \mathcal{D}_{G}$ is a braided tensor functor.

Proof. Since $\mathcal{E}$ is a Tannakian subcategory of $\mathcal{C}$, then we know that FPdim $\mathcal{E}=$ $q^{j}$, for some $1 \leq j \leq n-m$, and $\mathcal{E} \subseteq \mathcal{C}_{\text {nil }}=\mathcal{D}^{\prime}$ (see Remark 4.1 (i)). In particular, $\mathcal{E}$ is contained in the Müger center of $\mathcal{D}$, which coincides with $\mathcal{D} \cap \mathcal{D}^{\prime}$. This implies that $\mathcal{D}_{G}$ is a braided fusion category and the canonical functor $F: \mathcal{D} \rightarrow \mathcal{D}_{G}$ is a braided tensor functor, as claimed.

Suppose that $\mathrm{FPdim} \mathcal{E}=q^{j}, 1 \leq j \leq n-m$. Then $\mathrm{FPdim} \mathcal{D}_{G}=b q^{n-m-j}$. By Lemma 4.3, there is an action of the group $G$ on $\mathcal{D}_{G}$ by braided autoequivalences such that $\mathcal{D} \cong\left(\mathcal{D}_{G}\right)^{G}$.

Since $d$ is by assumption square-free and not divisible by $q$, then we may write $b=p_{1} \ldots p_{r}$, where $p_{1}, \ldots, p_{r}$ are pairwise distinct prime numbers and $p_{i} \neq q$, for all $1 \leq i \leq r$.

The fact that $\mathcal{D}_{G}$ is pointed implies that $\mathcal{D}_{G}$ contains unique fusion subcategories $\left(\mathcal{D}_{G}\right)_{p_{1}}, \ldots,\left(\mathcal{D}_{G}\right)_{p_{r}}$ and $\left(\mathcal{D}_{G}\right)_{q}$ such that $\operatorname{FPdim}\left(\mathcal{D}_{G}\right)_{p_{i}}=p_{i}$, $1 \leq i \leq r$, and $\mathrm{FPdim}\left(\mathcal{D}_{G}\right)_{q}=q^{n-m-j}$. Moreover, there is an equivalence of braided fusion categories

$$
\mathcal{D}_{G} \cong\left(\mathcal{D}_{G}\right)_{p_{1}} \otimes \cdots \otimes\left(\mathcal{D}_{G}\right)_{p_{r}} \otimes\left(\mathcal{D}_{G}\right)_{q} .
$$

The fusion subcategories $\left(\mathcal{D}_{G}\right)_{p_{i}}$ and $\left(\mathcal{D}_{G}\right)_{q}$ are clearly stable under the action of the group $G$, so that the equivariantizations $\mathcal{D}_{p_{i}}:=\left(\mathcal{D}_{G}\right)_{p_{i}}^{G}$ and $\mathcal{D}_{q}:=\left(\mathcal{D}_{G}\right)_{q}^{G}$ are fusion subcategories of $\mathcal{D}$ such that $\operatorname{FPdim} \mathcal{D}_{p_{i}}^{G}=p_{i} q^{j}$ and $\operatorname{FPdim} \mathcal{D}_{q}^{G}=q^{n-m}$.

A dimension argument shows that $\mathcal{D}=\mathcal{D}_{p_{1}} \vee \cdots \vee \mathcal{D}_{p_{r}} \vee \mathcal{D}_{q}$. Since $\mathcal{D}_{q}$ is nilpotent, there exists $n \geq 1$ such that $\mathcal{D}_{q}^{(n)} \cong$ Vect. In view of Lemma 2.2, this implies that $\mathcal{D}=\mathcal{D}^{(n)}$ is generated by $\mathcal{D}_{p_{i}}^{(n)}, 1 \leq i \leq r$. Then necessarily

$$
\mathcal{D}=\mathcal{D}_{p_{1}} \vee \cdots \vee \mathcal{D}_{p_{r}} .
$$

Lemma 4.4. $\mathrm{FPdim} \mathcal{D}_{p_{i_{1}}} \vee \cdots \vee \mathcal{D}_{p_{i_{s}}}=q^{j} p_{i_{1}} \ldots p_{i_{s}}$, for all pairwise distinct $1 \leq i_{1}, \ldots, i_{s} \leq r, s \geq 1$.

Proof. The proof is by induction on $s$. The claim is clear if $s=1$. Assume $s>1$. Notice that $\mathcal{E} \subseteq \cap_{i=1}^{r} \mathcal{D}_{p_{i}}$, so that $q^{j}$ divides $\operatorname{FPdim}\left(\mathcal{D}_{p_{i_{1}}} \vee \cdots \vee\right.$ $\left.\mathcal{D}_{p_{i_{s-1}}}\right) \cap \mathcal{D}_{p_{i_{s}}}$. By the inductive assumption, $\operatorname{FPdim}\left(\mathcal{D}_{p_{i_{1}}} \vee \cdots \vee \mathcal{D}_{p_{i_{s-1}}}\right) \cap \mathcal{D}_{p_{i_{s}}}$ divides $q^{j}$ and therefore $\operatorname{FPdim}\left(\mathcal{D}_{p_{i_{1}}} \vee \cdots \vee \mathcal{D}_{p_{i_{s-1}}}\right) \cap \mathcal{D}_{p_{i_{s}}}=q^{j}$. The identity follows by induction from [12, Corollary 3.12].

Lemma 4.5. (i) $\mathrm{FPdim} \mathcal{E}=q^{n-m}$.

(ii) The Tannakian subcategory $\mathcal{E}$ coincides with the Müger center of $\mathcal{D}$. In particular, $\mathcal{D}_{G}$ is non-degenerate. 
Proof. It follows from Lemma 4.4 that

$$
\operatorname{FPdim}\left(\mathcal{D}_{p_{1}} \vee \cdots \vee \mathcal{D}_{p_{r}}\right)=p_{1} \ldots p_{r} q^{j}=b q^{j} .
$$

Therefore $\mathrm{FPdim} \mathcal{D}=b q^{n-m}=\operatorname{FPdim}\left(\mathcal{D}_{p_{1}} \vee \cdots \vee \mathcal{D}_{p_{r}}\right)=b q^{j}$, which implies that $j=n-m$. This proves (i).

We now show (ii). Let $\mathcal{B}=\mathcal{D} \cap \mathcal{D}^{\prime}$ be the Müger center of $\mathcal{D}$. By Lemma 4.3. $\mathcal{E} \subseteq \mathcal{B}$. On the other hand, $\mathrm{FP} \operatorname{dim} \mathcal{B}$ divides $\mathrm{FPdim} \mathcal{D}=b q^{n-m}$ and $\mathrm{FP} \operatorname{dim} \mathcal{D}^{\prime}=a q^{m}$, whence $\mathrm{FP} \operatorname{dim} \mathcal{B}$ divides $q^{n-m}$. In view of part (i), this implies that $\mathcal{E}=\mathcal{B}$, as claimed.

We shall denote by $\rho: \underline{G} \rightarrow \operatorname{Aut}_{b r} \mathcal{D}_{G}$ the action by braided autoequivalences of $\mathcal{D}_{G}$ such that $\mathcal{D} \cong\left(\mathcal{D}_{G}\right)^{G}$.

Let also $\left(\operatorname{Irr} \mathcal{D}_{G}\right)_{p_{i}}$ be the set of isomorphism classes of simple objects of $\left(\mathcal{D}_{G}\right)_{p_{i}}, 1 \leq i \leq r$. Thus $\left(\operatorname{Irr} \mathcal{D}_{G}\right)_{p_{i}}$ is a cyclic group of order $p_{i}$ and the action $\rho$ induces an action of $G$ by group automorphisms on $\left(\operatorname{Irr} \mathcal{D}_{G}\right)_{p_{i}}$, for all $1 \leq i \leq r$.

Lemma 4.6. The action of $G$ on $\left(\operatorname{Irr} \mathcal{D}_{G}\right)_{p_{i}}$ is not trivial, for all $1 \leq i \leq r$.

Proof. Suppose on the contrary that there exists some $1 \leq i \leq r$ such that the action of $G$ on $\left(\operatorname{Irr} \mathcal{D}_{G}\right)_{p_{i}}$ is trivial, that is, $\rho^{g}(Y) \cong Y$, for all simple objects $Y$ of $\left(\mathcal{D}_{G}\right)_{p_{i}}$. Since $\mathcal{D}_{G}$ is pointed, then every such simple object $Y$ is invertible, and thus $Y^{*} \cong Y^{-1}$.

Let $X$ be a simple object of $\mathcal{D}_{p_{i}}$ and let $Y$ be a simple constituent of $F(X)$ in $\left(\mathcal{D}_{G}\right)_{p_{i}}$. Since the action of $G$ on $\left(\operatorname{Irr} \mathcal{D}_{G}\right)_{p_{i}}$ is trivial, then $F(X) \cong Y^{(d)}$ [4, Proposition 2.1]. Therefore

$$
F\left(X \otimes X^{*}\right) \cong F(X) \otimes F(X)^{*} \cong Y^{(d)} \otimes\left(Y^{-1}\right)^{(d)} \cong \mathbf{1}^{\left(d^{2}\right)} .
$$

This implies that the essential image of $\left(\mathcal{D}_{p_{i}}\right)_{a d}$ under the functor $F$ is the trivial subcategory $\langle\mathbf{1}\rangle$ of $\left(\mathcal{D}_{G}\right)_{p_{i}}$. Hence $\left(\mathcal{D}_{p_{i}}\right)_{a d} \subseteq \mathcal{E}$.

Since $\mathcal{D}=\mathcal{D}_{a d}=\left(\mathcal{D}_{p_{1}}\right)_{a d} \vee \cdots \vee\left(\mathcal{D}_{p_{r}}\right)_{a d}$, and $\mathcal{E} \subseteq \mathcal{D}_{p_{l}}$, for all $l=1, \ldots, r$, we obtain that

$$
\begin{aligned}
\mathcal{D} & =\left(\mathcal{D}_{p_{1}}\right)_{a d} \vee \cdots \vee\left(\mathcal{D}_{p_{i-1}}\right)_{a d} \vee\left(\mathcal{D}_{p_{i+1}}\right)_{a d} \cdots \vee\left(\mathcal{D}_{p_{r}}\right)_{a d} \\
& \subseteq \mathcal{D}_{p_{1}} \vee \cdots \vee \mathcal{D}_{p_{i-1}} \vee \mathcal{D}_{p_{i+1}} \cdots \vee \mathcal{D}_{p_{r}}
\end{aligned}
$$

This contradicts Lemma 4.4, since FPdim $\mathcal{D}=b q^{n}$ while

$$
\operatorname{FPdim} \mathcal{D}_{p_{1}} \vee \cdots \vee \mathcal{D}_{p_{i-1}} \vee \mathcal{D}_{p_{i+1}} \ldots \vee \mathcal{D}_{p_{r}}=p_{1} \ldots p_{i-1} p_{i+1} \ldots p_{r} q^{n} .
$$

This contradiction shows that the action of $G$ on $\left(\operatorname{Irr} \mathcal{D}_{G}\right)_{p_{i}}$ must be nontrivial, for all $1 \leq i \leq r$, as claimed.

We next combine the previous lemmas to prove the main result of this section.

Theorem 4.7. Let $q$ be an odd prime number and let $d \geq 1$ be a square-free integer. Suppose that $\mathcal{C}$ is an $A S F$ modular category such that $\mathrm{FPdim} \mathcal{C}=$ $d q^{n}, n \geq 0$. Then $\mathcal{C}$ is integral and nilpotent. 
Proof. Since $q$ is odd and $d$ is square-free, $\mathrm{FPdim} \mathcal{C}$ is not divisible by 4 , hence $\mathcal{C}$ is integral by Corollary 3.2 .

Suppose that $\mathcal{C}$ is not nilpotent. We may assume that $d$ is not divisible by $q$ and $n \geq 1$.

By Lemmas 4.3, 4.5 and 4.6, the Müger center $\mathcal{E}$ of the category $\mathcal{D}=$ $\left(\mathcal{C}_{\text {nil }}\right)^{\prime}$ is a Tannakian subcategory, $\mathcal{E} \cong \operatorname{Rep} G$, where $G$ is a group of order $q^{n-m}$. Furthermore, the de-equivariantization $\mathcal{D}_{G}$ is a non-degenerate braided fusion category of dimension $d$ and the action by braided autoequivalences of the group $G$ on $\mathcal{D}_{G}$ induces a nontrivial action on $\left(\operatorname{Irr} \mathcal{D}_{G}\right)_{p_{i}}$, for all $1 \leq i \leq r$. Then $q$ must divide $p_{i}-1$, for all $1 \leq i \leq r$. In particular, the primes $p_{i}$ and therefore also $b$ must be odd. Notice that the categories $\left(\mathcal{D}_{G}\right)_{p_{i}}, 1 \leq i \leq r$, in the decomposition (4.2) of $\mathcal{D}_{G}$ are also non-degenerate (see Remark 2.1).

Let $p=p_{i}, 1 \leq i \leq r$. Non-degenerate braided fusion categories of dimension $p$ are classified by metric groups $\left(\mathbb{F}_{p}, \phi\right)$, where $\mathbb{F}_{p}$ denotes the field with $p$ elements and $\phi$ is a non-degenerate quadratic from on $\mathbb{F}_{p}$. Moreover, the quadratic form $\phi$ is one of the following:

$$
\phi_{1}(a)=\zeta^{a^{2}}, \quad \text { or } \quad \phi_{2}(a)=\zeta^{c a^{2}},
$$

where $\zeta \in k$ is some primitive $p$ th. root of unity and $c \in \mathbb{F}_{p}^{\times}$is a quadratic nonresidue. See [12, Proposition A.6].

The action by braided autoequivalences $\rho: \underline{G} \rightarrow \operatorname{Aut}_{b r}\left(\mathcal{D}_{G}\right)_{p}$ corresponds to an action $\rho: G \rightarrow \operatorname{Aut}\left(\mathbb{F}_{p}\right) \cong \mathbb{F}_{p}^{\times}$of $G$ on $\mathbb{F}_{p}$ by group automorphisms preserving the form $\phi$. In view of the possibilities (4.4), the nontrivial action $\rho$ must satisfy

$$
\rho(g)= \pm 1(\bmod p)
$$

for all $g \in G$. This implies that $G$ is a 2-group, that is, $q=2$. Thus we obtain that every non-degenerate braided fusion category of FrobeniusPerron dimension $d q^{n}$ such that $q$ is odd is nilpotent, as claimed.

One of the main results in [11] shows that an integral nilpotent modular category is group-theoretical. As a consequence of Theorem 4.7 we obtain:

Corollary 4.8. Let $q$ be an odd prime number and let $d \geq 1$ be a squarefree integer. Then every ASF modular category of dimension $d q^{n}, n \geq 0$, is group-theoretical.

Remark 4.9. Let $\mathcal{C}$ be an ASF modular category. Suppose as before that FPdim $\mathcal{C}=d q^{n}$, where $q>2, n \geq 1$ and $d$ is a square-free natural number not divisible by $q$. Let $d=p_{1} \ldots p_{r}$ be the decomposition of $d$ as a product of distinct prime numbers. Since $\mathcal{C}$ is nilpotent, then

$$
\mathcal{C} \cong \mathcal{C}_{p_{1}} \otimes \cdots \otimes \mathcal{C}_{p_{r}} \otimes \mathcal{C}_{q^{n}},
$$

where $\mathcal{C}_{p_{i}}$ is a (necessarily pointed) modular category of Frobenius-Perron dimension $p_{i}, 1 \leq i \leq r$, and $\mathcal{C}_{q^{n}}$ is a modular category of Frobenius-Perron dimension $q^{n}$ [11, Theorem 1.1]. 
Pointed modular categories of prime dimension $p$ are classified by metric groups $\left(\mathbb{F}_{p}, \phi\right)$, where $\mathbb{F}_{p}$ denotes the field with $p$ elements and $\phi$ is a nondegenerate quadratic from on $\mathbb{F}_{p}$ as in (4.4).

Therefore the problem of giving an explicit parameterization of all possible ASF modular categories $\mathcal{C}$ under the mentioned restrictions reduces to the explicit determination of modular categories of odd prime power FrobeniusPerron dimension. This problem is beyond the scope of this paper, and will be postponed for future consideration.

However, in view of [25, Theorem 5.11 and Proposition 6.7 (i)], we can say that a modular category of Frobenius-Perron dimension $q^{n}$ (which is necessarily group-theoretical [11]) can be described by means of a factorization $G=K H$ of a finite $q$-group $G$ into mutually centralizing normal subgroups $H$ and $K$, together with a 3 -cocycle $\omega$ on $G$ and a $G$-invariant $\omega$-bicharacter $b: K \times H \rightarrow k^{\times}$such that a certain symmetric bicharacter associated to $b$ is non-degenerate on $H \cap K$.

Our next result, Corollary 4.13, is an application of Theorem 4.7 in the dimension $d q^{4}$ case. We shall use the following lemma:1]:

Lemma 4.10. Let $q$ be a prime number. Then the Drinfeld center of a fusion category of dimension $q^{2}$ is a pointed modular category.

Proof. Suppose that $G$ is any finite group and $\omega \in Z^{3}\left(G, k^{\times}\right)$is a 3-cocycle on $G$. Then the Drinfeld center of the pointed fusion category Vect ${ }_{G}^{\omega}$ is equivalent as a braided fusion category to the category $\operatorname{Rep}\left(D^{\omega} G\right)$ of finite dimensional representations of the twisted quantum double $D^{\omega} G$ [20].

Assume $G$ is abelian. Then the fusion category $\operatorname{Rep}\left(D^{\omega} G\right)$ is pointed if and only if the class of the 3-cocycle $\omega$ belongs to the subgroup $H^{3}\left(G, k^{\times}\right)_{a b}$ of $H^{3}\left(G, k^{\times}\right)$, defined as $H^{3}\left(G, k^{\times}\right)_{a b}=\cap_{x \in G} D_{x}$, where for all $x \in G, D_{x}$ : $H^{3}\left(G, k^{\times}\right) \rightarrow H^{2}\left(G, k^{\times}\right)$is the group homomorphism that maps the class of a 3-cocycle $\omega \in Z^{3}\left(G, k^{\times}\right)$to the class of the 2-cocycle $\omega_{x} \in Z^{2}\left(G, k^{\times}\right)$ defined in the form

$$
\omega_{x}(g, h)=\frac{\omega(x, g, h) \omega(g, h, x)}{\omega(g, x, h)},
$$

for all $g, h \in G$. See [21, Corollary 3.6].

Let now $\mathcal{C}$ be a fusion category of dimension $q^{2}$. Then $\mathcal{C}$ is pointed and thus equivalent to the category $\operatorname{Vect}_{G}^{\omega}$ for some group $G$ of order $q^{2}$ and $\omega \in Z^{3}\left(G, k^{\times}\right)$. The group $G$ must be isomorphic to one of the groups $G_{1}=\left\langle c: c^{q^{2}}=1\right\rangle \cong \mathbb{Z}_{q^{2}}$ or $G_{2}=\left\langle a, b: a^{q}=b^{q}=a b a^{-1} b^{-1}=1\right\rangle \cong \mathbb{Z}_{q} \times \mathbb{Z}_{q}$.

The group $H^{3}\left(G_{1}, k^{\times}\right) \cong \mathbb{Z}_{q^{2}}$ is generated by the class of the 3-cocycle $\omega_{I}$ defined in the form

$$
\omega_{I}\left(c^{i}, c^{i^{\prime}}, c^{i^{\prime \prime}}\right)=\xi^{i\left[\frac{i^{\prime}+i^{\prime \prime}}{q^{2}}\right]},
$$

\footnotetext{
${ }^{1}$ Lemma 4.10 was motivated by a question of the referee, that we acknowledge with thanks; it improves a version of Corollary 4.13 obtained in a previous version of this paper.
} 
for all $0 \leq i, i^{\prime}, i^{\prime \prime} \leq q^{2}-1$, where $\xi \in k^{\times}$is a primitive $q^{2}$ th root of unity and for each $m \in \mathbb{Q}$, the notation $[m]$ indicates the largest integer less than $m$.

Observe that the generator $\omega_{I}$ satisfies the symmetry condition

$$
\omega_{I}(x, g, h)=\omega_{I}(x, h, g),
$$

for all $x, g, h \in G_{1}$. This implies that for all $x \in G_{1}$ the class of the 2-cocycle $\omega_{x}$ is trivial in $H^{2}\left(G_{1}, k^{\times}\right)$. We thus obtain that $H^{3}\left(G_{1}, k^{\times}\right)=H^{3}\left(G_{1}, k^{\times}\right)_{a b}$ and therefore the category $\operatorname{Rep}\left(D^{\omega} G_{1}\right)$ is pointed for every 3-cocycle $\omega$ on $G_{1}$.

Regarding the group $G_{2}$, we have that $H^{3}\left(G_{2}, k^{\times}\right) \cong \mathbb{Z}_{q} \times \mathbb{Z}_{q} \times \mathbb{Z}_{q}$ is generated by the classes of the 3 -cocycles $\omega_{I}^{(1)}, \omega_{I}^{(2)}$ and $\omega_{I I}$ given, respectively, by the formulas

$$
\begin{aligned}
& \omega_{I}^{(1)}\left(a^{i} b^{j}, a^{i^{\prime}} b^{j^{\prime}}, a^{i^{\prime \prime}} b^{j^{\prime \prime}}\right)=\zeta^{i\left[\frac{i^{\prime}+i^{\prime \prime}}{q}\right]}, \\
& \omega_{I}^{(2)}\left(a^{i} b^{j}, a^{i^{\prime}} b^{j^{\prime}}, a^{i^{\prime \prime}} b^{j^{\prime \prime}}\right)=\zeta^{j\left[\frac{j^{\prime}+j^{\prime \prime}}{q}\right]}, \\
& \omega_{I I}\left(a^{i} b^{j}, a^{i^{\prime}} b^{j^{\prime}}, a^{i^{\prime \prime}} b^{j^{\prime \prime}}\right)=\zeta^{i\left[\frac{j^{\prime}+j^{\prime \prime}}{q}\right]},
\end{aligned}
$$

for all $0 \leq i, i^{\prime}, i^{\prime \prime}, j, j^{\prime}, j^{\prime \prime} \leq q-1$, where $\zeta \in k^{\times}$is a primitive $q$ th root of unity. See for instance [6, Section 2.3.2].

As in the case of $G_{1}$, we find that if $\omega$ is any of the generators $\omega_{I}^{(1)}, \omega_{I}^{(2)}$ or $\omega_{I I}$, then $\omega$ satisfies the symmetry condition (4.5) for all elements $x, g, h \in$ $G_{2}$. Then also in this case $H^{3}\left(G_{2}, k^{\times}\right)=H^{3}\left(G_{2}, k^{\times}\right)_{a b}$ and therefore the category $\operatorname{Rep}\left(D^{\omega} G_{2}\right)$ is pointed for every 3-cocycle $\omega$ on $G_{2}$. This finishes the proof of the lemma.

Lemma 4.11. Let $q$ be a prime number and let $\mathcal{C}$ be an integral modular category of dimension $q^{4}$. Then $\mathcal{C}$ is pointed.

Proof. Suppose on the contrary that $\mathcal{C}$ is not pointed. By [9, Lemma 3.4], $\left(\mathcal{C}_{a d}\right)_{p t}$ is a symmetric subcategory of dimension $q^{2}$. Since $\mathcal{C}$ is modular, the dimension of every non-invertible simple object is $q$. Hence, $\left(\mathcal{C}_{a d}\right)_{p t}=\mathcal{C}_{p t}$ is the unique fusion subcategory of dimension $q^{2}$.

Case $q=2 . \mathcal{C}$ is of type $(1,4 ; 2,3)$ and hence it is a modular category of rank 7. By [2, Theorem 5.8], it should be pointed, a contradiction.

Case $q>2$. In this case the symmetric category $\mathcal{C}_{p t}$ is necessarily Tannakian. By [22, Theorem 3.2], FPdim $\mathcal{C}_{p t}^{\prime}=q^{2}$. Since $\mathcal{C}_{p t}$ is the unique fusion subcategory of dimension $q^{2}$, we get $\mathcal{C}_{p t}^{\prime}=\mathcal{C}_{p t}$ and hence the subcategory $\mathcal{C}_{p t}$ is Lagrangian. It follows from [11, Theorem 4.5] that $\mathcal{C}$ is equivalent to the Drinfeld center of a fusion category of dimension $q^{2}$. Lemma 4.10 implies that $\mathcal{C}$ must be pointed, against the assumption. This contradiction finishes the proof of the lemma.

Remark 4.12. Observe that if $q$ is an odd prime number, then every modular category $\mathcal{C}$ of Frobenius-Perron dimension $q^{4}$ is integral and therefore 
pointed, by Lemma 4.11. On the other hand, the Drinfeld center of an Ising category provides an example of a non-pointed (and not integral) modular category of Frobenius-Perron dimension 16.

Corollary 4.13. Let $\mathcal{C}$ be a modular category of Frobenius-Perron dimension $d q^{4}$, where $q$ is an odd prime number and $d$ is a square-free integer not divisible by $q$. Then $\mathcal{C}$ is pointed.

Proof. Let $d=p_{1} \ldots p_{s}$, where $p_{1}, \ldots, p_{s}$ are pairwise distinct prime numbers, distinct from $q$. By Theorem 4.7, $\mathcal{C}$ is integral and nilpotent. Therefore, in view of [11, Theorem 1.1], $\mathcal{C} \cong \mathcal{C}_{q^{4}} \otimes \mathcal{C}_{p_{1}} \otimes \cdots \otimes \mathcal{C}_{p_{s}}$, where $\mathcal{C}_{t}$ is a modular category of dimension $t$ (see Remark 2.1). In particular, the categories $\mathcal{C}_{p_{1}}, \ldots, \mathcal{C}_{p_{s}}$ are pointed. It follows from Lemma 4.11 that $\mathcal{C}_{q^{4}}$ is also pointed, which implies the statement.

\section{Structure of a STRictly Weakly integral ASF modular CATEGORY}

Let $\mathcal{D}$ be a fusion category. If $\mathcal{D}$ is not pointed and $X \otimes Y$ is a direct sum of invertible objects, for all non-invertible simple objects $X, Y \in \mathcal{D}$, then $\mathcal{D}$ is called a generalized Tambara-Yamagami fusion category. Generalized Tambara-Yamagami fusion categories were classified in [19], up to equivalence of tensor categories. Recently, they have been further studied in [28]. In particular, modular generalized Tambara-Yamagami fusion categories were classified in terms of Ising modular categories and pointed modular categories.

Proposition 5.1. Let $\mathcal{D}$ be a weakly integral fusion category such that $\operatorname{FPdim}\left(\mathcal{D}_{a d}\right)=2$. Then $\mathcal{D}$ is a generalized Tambara-Yamagami fusion category. In particular, if $\mathcal{D}$ is modular, then $\mathcal{D} \cong \mathcal{I} \otimes \mathcal{B}$, where $\mathcal{I}$ is an Ising fusion category and $\mathcal{B}$ is a pointed modular category.

Proof. Since $\operatorname{FPdim}\left(\mathcal{D}_{a d}\right)=2$, then $\mathcal{D}$ is not pointed. Let $\mathcal{D}=\oplus_{g \in \mathcal{U}(\mathcal{D})} \mathcal{D}_{g}$ be the universal grading of $\mathcal{D}$. Then $\operatorname{FPdim}\left(\mathcal{D}_{g}\right)=2$ for all $g \in \mathcal{U}(\mathcal{D})$. Since $\mathcal{D}$ is weakly integral and not pointed, the FP dimension of every simple object is a square root of some positive integer. Hence, every component $\mathcal{D}_{g}$ of the universal grading either contains two non-isomorphic invertible objects, or it contains a unique $\sqrt{2}$-dimensional simple object. Thus we get that the Frobenius-Perron dimension of any simple object of $\mathcal{D}$ is 1 or $\sqrt{2}$. Hence $\mathcal{C}_{\text {int }}$ is a pointed fusion category.

Considering the dimensional grading $\mathcal{D}=\oplus_{h \in E} \mathcal{D}_{h}$, we get that the order of $E$ is 2 . We also get that $\mathcal{D}_{0}$ contains 1-dimensional simple objects, and $\mathcal{D}_{1}$ contains $\sqrt{2}$-dimensional simple objects. This implies that $\mathcal{D}$ is a $\mathbb{Z}_{2^{-}}$ extension of a pointed fusion category. Let $X, Y \in \mathcal{D}_{1}$ be simple objects. Then $X \otimes Y \in \mathcal{D}_{0}$ is a direct sum of invertible objects. This proves that $\mathcal{D}$ is a generalized Tambara-Yamagami fusion category.

If $\mathcal{D}$ is modular then [28, Theorem 5.5] (see also [8, Theorem 3.4]) shows that $\mathcal{D} \cong \mathcal{I} \otimes \mathcal{B}$ as described. 
Let $\mathcal{C}$ be an ASF modular category of Frobenius-Perron dimension $d q^{n}$. In the case when $\mathcal{C}$ is integral, the structure of $\mathcal{C}$ has been obtained in [9, Corollary 4.2]: $\mathcal{C}$ is either pointed, or equivalent to a $G$-equivariantization of a nilpotent fusion category of nilpotency class 2 , where $G$ is a $q$-group. Note in addition that if $\mathcal{C}$ is strictly weakly integral, then $q=2$, by Corollary 3.2 ,

For the rest of this section, $\mathcal{C}$ will denote a weakly integral ASF modular category of Frobenius-Perron dimension $d 2^{n}$.

Lemma 5.2. The dimension of every simple object of $\mathcal{C}_{a d}$ is a power of 2.

Proof. Let $X$ be a simple object of $\mathcal{C}_{a d}$. It is known that $\mathcal{C}_{a d} \subseteq \mathcal{C}_{\text {int }}$ is an integral fusion subcategory of $\mathcal{C}$. Hence $\mathrm{FPdim} X$ is an integer. On the other hand, $X$ is also a simple object of $\mathcal{C}$. It follows that FPdim $X^{2}$ divides $\mathrm{FP} \operatorname{dim} \mathcal{C}=d 2^{n}$ by [16, Theorem 2.11]. Hence, $\mathrm{FPdim} X$ is a power of 2.

The following lemma is a special case of [3, Lemma 4.1]:

Lemma 5.3. If $|\mathcal{U}(\mathcal{C})|$ is divisible by $2^{n}$ then $\mathcal{C}$ is pointed.

Lemma 5.4. Assume that $\mathcal{C}$ is not pointed. Then $\left(\mathcal{C}_{a d}\right)_{p t}$ is not trivial.

Proof. By Lemma 5.3, $|\mathcal{U}(\mathcal{C})|$ is not divisible by $2^{n}$. Hence FPdim $\mathcal{C}_{a d}$ is divisible by 2 . By Lemma 5.2, the dimension of every simple object of $\mathcal{C}_{a d}$ is a power of 2 . Let $1,2,2^{2}, \cdots, 2^{t}$ be all possible dimensions of simple objects of $\mathcal{C}_{a d}$, and let $a_{0}, a_{1}, a_{2}, \cdots, a_{t}$ be the number of non-isomorphic simple objects of these dimensions. Then we have $a_{0}+a_{1} 2^{2}+a_{2} 2^{4}+\cdots+a_{t} 2^{2 t}=$ FPdim $\mathcal{C}_{a d}$. Since the right hand side is divisible by 2 , we get that $a_{0}$ is also divisible by 2. This shows that $\left(\mathcal{C}_{a d}\right)_{p t}$ is not trivial.

Proposition 5.5. Assume that $\mathcal{C}$ is not pointed and $\mathrm{FPdim} \mathcal{C}_{a d}>2$. Then $\mathcal{C}$ contains a non-trivial Tannakian subcategory.

Proof. By Lemma 5.4 and [18, Lemma 2.4], $\left(\mathcal{C}_{a d}\right)_{p t}$ is a non-trivial symmetric fusion category. If $\mathrm{FPdim}\left(\mathcal{C}_{a d}\right)_{p t}>2$ then $\left(\mathcal{C}_{a d}\right)_{p t}$ contains a non-trivial Tannakian subcategory with Frobenius-Perron dimension $\operatorname{FPdim}\left(\mathcal{C}_{a d}\right)_{p t} / 2$ (see the exposition in Section [2.3). Hence we may assume in the rest that $\operatorname{FPdim}\left(\mathcal{C}_{a d}\right)_{p t}=2$.

Since we have assumed that $\mathrm{FP} \operatorname{dim} \mathcal{C}_{a d}>2, \mathcal{C}_{a d}$ contains non-invertible simple objects. Let $\delta$ be the unique non-trivial invertible simple object in $\left(\mathcal{C}_{a d}\right)_{p t}$. Then $\delta$ appears in the decomposition of $X \otimes X^{*}$ for every noninvertible $X \in \operatorname{Irr}\left(\mathcal{C}_{a d}\right)$. To see this, we just notice that the Frobenius-Perron dimension of every non-invertible simple object of $\mathcal{C}_{a d}$ is a power of 2 , by Lemma 5.2. Therefore, we have

$$
\delta \otimes X \cong X, \quad X \in \operatorname{Irr}\left(\mathcal{C}_{a d}\right) /\{1, \delta\} .
$$

Since $\delta \in \mathcal{C}_{p t}=\mathcal{C}_{a d}^{\prime}$, it follows from [23, Lemma 5.4] that $\left(\mathcal{C}_{a d}\right)_{p t}$ cannot be the category sVect of super vector spaces. Hence, $\left(\mathcal{C}_{a d}\right)_{p t}$ must be a Tannakian subcategory. 
Theorem 5.6. Let $\mathcal{C}$ be a strictly weakly integral ASF modular category. Then $\mathcal{C}$ fits into one of the following classes:

(1) $\mathcal{C}$ is equivalent to a Deligne tensor product $\mathcal{I} \otimes \mathcal{B}$, where $\mathcal{I}$ is an Ising fusion category and $\mathcal{B}$ is a pointed modular category.

(2) $\mathcal{C}$ is equivalent to a $G$-equivariantization of a braided $G$-crossed $f u$ sion category $\oplus_{g \in G} \mathcal{D}_{g}$, where $G$ is a 2 -group and $\mathcal{D}_{e}$ is a pointed modular category.

(3) $\mathcal{C}$ is equivalent to a $G$-equivariantization of a braided $G$-crossed fusion category $\oplus_{g \in G} \mathcal{D}_{g}$, where $G$ is a 2-group and $\mathcal{D}_{e} \cong \mathcal{I} \otimes \mathcal{B}$, where $\mathcal{I}$ is an Ising fusion category and $\mathcal{B}$ is a pointed modular category.

Proof. Suppose that FPdim $\mathcal{C}_{a d}=2$. Then (1) holds true by Proposition 5.1. In the rest of our proof, we consider the case where FPdim $\mathcal{C}_{a d}>2$.

By Proposition 5.5, $\mathcal{C}$ has a non-trivial Tannakian subcategory. Let $\mathcal{D} \cong$ $\operatorname{Rep}(G)$ be a proper maximal Tannakian subcategory of $\mathcal{C}$, and let $\mathcal{D}^{\prime}$ be its Müger centralizer in $\mathcal{C}$. Then $\mathcal{D}$ is the Müger center of $\mathcal{D}^{\prime}$. Let $\mathcal{E}=\left(\mathcal{D}^{\prime}\right)_{G}$ be the de-equivariantization of $\mathcal{D}^{\prime}$ by $\operatorname{Rep}(G)$. It is non-degenerate by 16 , Remark 2.3]. Since the dimension of $\mathcal{D}$ is a power of 2 and FPdim $\mathcal{E}=$ $\mathrm{FP} \operatorname{dim} \mathcal{C} / 4$, we know that $\mathcal{E}$ is also an ASF modular category.

Let $\mathcal{C}_{G}$ be the de-equivariantization of $\mathcal{C}$ by $\operatorname{Rep}(G)$. Then $\mathcal{C}_{G}=\oplus_{g \in G}\left(\mathcal{C}_{G}\right)_{g}$ has a faithful $G$-grading and the neutral component $\left(\mathcal{C}_{G}\right)_{e}$ is non-degenerate. By [12, Proposition 4.56], $\left(\mathcal{C}_{G}\right)_{e} \cong\left(\mathcal{D}^{\prime}\right)_{G}=\mathcal{E}$.

The fusion category $\mathcal{E}$ is the core of $\mathcal{C}$ in the sense of [12, section 5.4]. It is a weakly anisotropic braided fusion category by [12, Corollary 5.19]. By [12, Corollary 5.29], $\mathcal{E}_{p t} \cap\left(\mathcal{E}_{p t}\right)^{\prime}$ is either equivalent to Vect, or to the category sVect of super vector spaces.

If $\mathcal{E}_{p t} \cap\left(\mathcal{E}_{p t}\right)^{\prime} \cong$ Vect then $\mathcal{E}$ is pointed. In fact, if $\mathcal{E}$ is not pointed then the proof of Proposition 5.5 shows that $\mathcal{E}_{p t} \cap\left(\mathcal{E}_{p t}\right)^{\prime}=\mathcal{E}_{p t} \cap \mathcal{E}_{a d}=\left(\mathcal{E}_{a d}\right)_{p t}$ contains a non-trivial $G$-stable Tannakian subcategory. In this case, $\mathcal{C} \cong\left(\mathcal{C}_{G}\right)^{G}$ fits into the second class.

If $\mathcal{E}_{p t} \cap\left(\mathcal{E}_{p t}\right)^{\prime} \cong$ sVect then $\left(\mathcal{E}_{a d}\right)_{p t}=\mathcal{E}_{p t} \cap \mathcal{E}_{a d}=\mathcal{E}_{p t} \cap\left(\mathcal{E}_{p t}\right)^{\prime}$ has FrobeniusPerron dimension 2. By the proof of Proposition 5.5, we get that FPdim $\mathcal{E}_{a d}=$ 2. Hence Proposition 5.1 shows that $\mathcal{E}$ is equivalent to a Deligne tensor product $\mathcal{I} \otimes \mathcal{B}$, where $\mathcal{I}$ is an Ising fusion category and $\mathcal{B}$ is a modular pointed category. In this case, $\mathcal{C} \cong\left(\mathcal{C}_{G}\right)^{G}$ fits into the third class.

Remark 5.7. Let $n \geq 2$. Examples of strictly weakly integral ASF modular categories of Frobenius-Perron dimension $d 2^{2 n}, d \geq 1$, which are not in the class described in Theorem 5.6 (1), are provided by the tensor products

$$
\mathcal{I}_{1} \otimes \cdots \otimes \mathcal{I}_{n} \otimes \mathcal{B}
$$

where $\mathcal{I}_{1}, \ldots, \mathcal{I}_{n}$ are Ising modular categories and $\mathcal{B}$ is a pointed modular category of dimension $d$.

Consider the case where $n=2$ and $\mathcal{B}$ is trivial, that is, we have a tensor product $\mathcal{C}=\mathcal{I}_{1} \otimes \mathcal{I}_{2}$ of two Ising modular categories. It follows from [12, 
Lemma B.24] that $\mathcal{C}$ is equivalent to a $\mathbb{Z}_{2}$-equivariantization of a braided $\mathbb{Z}_{2^{-}}$ crossed fusion category whose trivial homogeneous component is a pointed modular category corresponding to a certain metric group of order 4 ; hence $\mathcal{C}$ belongs to the class described in Theorem $5.6(2)$.

\section{ACKNOWLEDGements}

The first author was partially supported by the Fundamental Research Funds for the Central Universities (KYZ201564), the Natural Science Foundation of China (11201231) and the Qing Lan Project. The second author was partially supported by CONICET and SeCYT-UNC

\section{REFERENCES}

[1] P. Bruillard, C. Galindo, S.-M. Hong, Y. Kashina, et al., Classification of integral modular categories of Frobenius-Perron dimension $p q^{4}$ and $p^{2} q^{2}$, Canad. Math. Bull. 57 (4) (2014) 721-734.

[2] P. Bruillard, C. Galindo, S.-H. Ng, J. Y. Plavnik, E. C. Rowell, Z. Wang, On the classification of weakly integral modular categories, J. Pure Appl. Algebra 220 (6) (2016) $2364-2388$.

[3] P. Bruillard, J. Y. Plavnik, E. C. Rowell, Modular categories of dimension $p^{3} m$, with $m$ square-free, to appear in Proc. Amer. Math. Soc., preprint arXiv: 1609.04896.

[4] S. Burciu, S. Natale, Fusion rules of equivariantizations of fusion categories, J. Math. Phys. 54 (1) (2013) 013511.

[5] A. Davydov, M. Müger, D. Nikshych, V. Ostrik, The Witt group of non-degenerate braided fusion categories, J. Reine Angew. Math. 2013 (677) (2013) 135-177.

[6] M. D. F. de Wild Propitius, Topological Interactions in Broken Gauge Theories, Ph. D. Thesis, University of Amsterdam, 1995. Preprint arXiv:hep-th/9511195.

[7] R. Dijkgraaf, V. Pasquier and P. Roche, Quasi-quantum groups related to orbifold models In: Proc. Modern Quantum Field Theory, Tata Institute, Bombay (1990), 375-383.

[8] J. Dong, Braided $\mathbb{Z}_{q}$-extensions of pointed fusion categories, Proc. Amer. Math. Soc. 145 (3) (2017) 995-1001.

[9] J. Dong, L. Li, L. Dai, Integral almost square-free modular categories, J. Algebra Appl. 16 (5) (2017) 1750104, 14 pages.

[10] J. Dong, H. Tucker, Integral modular categories of Frobenius-Perron dimension $p q^{n}$, Algebr. Represent. Theor. 19 (2016) 33-46.

[11] V. Drinfeld, S. Gelaki, D. Nikshych, V. Ostrik, Group-theoretical properties of nilpotent modular categories, preprint arXiv:0704.0195.

[12] V. Drinfeld, S. Gelaki, D. Nikshych, V. Ostrik, On braided fusion categories I, Selecta Math., New Ser. 16 (1) (2010) 1-119.

[13] P. Etingof, S. Gelaki, Some properties of finite-dimensional semisimple Hopf algebras, Math. Res. Lett. 5 (2) (1998) 191-197.

[14] P. Etingof, S. Gelaki, V. Ostrik, Classification of fusion categories of dimension $p q$, Int. Math. Res. Not. 2004 (57), (2004) 3041-3056.

[15] P. Etingof, D. Nikshych, V. Ostrik, On fusion categories, Ann. Math. 162 (2) (2005) 581-642.

[16] P. Etingof, D. Nikshych, V. Ostrik, Weakly group-theoretical and solvable fusion categories, Adv. Math. 226 (1) (2011) 176-205.

[17] S. Gelaki, D. Nikshych, Nilpotent fusion categories, Adv. Math. 217 (3) (2008) 10531071. 
[18] M. E. González, S. Natale, On fusion rules and solvability of a fusion category, J. Group Theory 20 (1) (2017) 133-167.

[19] J. Liptrap, Generalized Tambara-Yamagami categories, preprint arXiv:1002.3166.

[20] S. Majid, Quantum double for quasi-Hopf algebras, Lett. Math. Phys. 45 (1998), 1-9.

[21] G. Mason, S.-H. Ng, Group Cohomology and Gauge Equivalence Classes of some Twisted Quantum Doubles, Trans. Amer. Math. Soc. 353 (2001), 3465-3509.

[22] M. Müger, On the structure of modular categories, Proc. London Math. Soc. 87 (02) (2003) 291-308.

[23] M. Müger, Galois theory for braided tensor categories and the modular closure, Adv. Math. 150 (2) (2000) 151-201.

[24] M. Müger, Galois extensions of braided tensor categories and braided crossed Gcategories, J. Algebra 277 (1) (2004) 256-281.

[25] D. Naidu, D. Nikshych, S. Witherspoon, Fusion subcategories of representation categories of twisted quantum doubles of finite groups, Internat. Math. Res. Notices 2009 (22) (2009) 4183-4219.

[26] D. Naidu, E. C. Rowell, A finiteness property for braided fusion categories, Algebr. Represent. Theory. 14 (5) (2011), 837-855.

[27] S. Natale, On group theoretical Hopf algebras and exact factorizations of finite groups, J. Algebra 270 (1) (2003) 199-211.

[28] S. Natale, Faithful simple objects, orders and gradings of fusion categories, Algebr. Geom. Topol. 13 (2013) 1489-1511.

[29] S. Natale, On weakly group-theoretical non-degenerate braided fusion categories, J. Noncommut. Geom. 8 (4) (2014) 1043-1060.

(Jingcheng Dong) College of Engineering, Nanjing Agricultural University, NANJING 210031, ChinA

E-mail address: dongjc@njau.edu.cn

(Sonia Natale) Facultad de Matemática, Astronomía y Física. Universidad Nacional de Córdoba. Ciem - COniCet. Ciudad Universitaria. (5000) Córdoba, Argentina

E-mail address: natale@famaf.unc.edu.ar

URL: http://www.famaf.unc.edu.ar/ natale 